\title{
Overview of Wood Plastic Composites: Focusing on Use of Bio-based Plastics and Co-extrusion Technique ${ }^{1}$
}

\author{
Birm-June $\mathrm{Kim}^{2, \dagger}$
}

\begin{abstract}
Wood filler is a porous and anisotropic material having different size, shape, and aspect ratio. The use of wood fillers such as wood particle, wood flour, and wood pulp in wood plastic composites (WPCs) are growing rapidly because these wood fillers give improved strength and stiffness to WPCs. However, the wood fillers have originally poor compatibility with plastic matrix affecting the mechanical properties of WPCs. Therefore, to improve compatibility between wood and plastic, numbers of physical and chemical treatments were investigated. While the various treatments led to improved performances in WPC industries using petroleum-based plastics, full biodegradation is still issues due to increased environmental concerns. Hence, bio-based plastics such as polylactide and polyhydroxybutyrate having biodegradable characteristics are being applied to WPCs, but relatively expensive prices of existing bio-based plastics prevent further uses. As conventional processing methods, extrusion, injection, and compression moldings have been used in WPC industries, but to apply WPCs to engineered or structural places, new processing methods should be developed. As one system, co-extrusion technique was introduced to WPCs and the co-extruded WPCs having core-shell structures make the extended applications of WPCs possible.
\end{abstract}

Keywords : wood filler, wood-plastic composites, treatment, bio-based plastics, co-extrusion

\section{INTRODUCTION}

Wood has been used as a reinforcing filler for wood-plastic composites (WPCs) because of its good properties such as light weight, dimensional stability, high stiffness, and low cost (Bledzki et al. 1998). However, wood fibers with hydrophilic characteristics poorly adhere to hydrophobic plastic matrix leading to low performances. Hence, to improve compatibility between them, a large number of physical and/or chemical treatments have been studied
(Bledzki et al. 1998; $\mathrm{Lu}$ and $\mathrm{Wu}$ 2000; Borysiak 2013). Through these studies, progresses in WPC properties have been reported.

The property improvements through various treatments have extended the market for WPCs and made the WPCs as reliable alternatives to conventional synthetic fiber reinforced composites. However, in these days, the increased concern for environmental contaminations and petroleum crisis from oil depletion has required new sustainable green plastic materials in WPCs (Mohanty et al. 2002; John and Thomas

1 Date Received February 24, 2014, Date Accepted April 27, 2014

2 Department of Forest Products and Biotechnology, Kookmin University, Seoul 136-702, Korea

† Corresponding author : Birm-June Kim (e-mail: bjkim3@kookmin.ac.kr) 
2008). Hence, bio-based plastics from renewable feedstock have attracted increased attentions from the industries and manufacturers seeking to replace petroleum-based plastics (Clemons 2002). Polylactide and polyhydroxybutyrate are representative bio-based plastics and their uses are accordingly growing with the development of new biodegradable and bio-based plastics.

Since WPCs were introduced in market, the research trends for the WPCs have been transformed from material to process. Extrusion, injection, and compression moldings have been conventionally used as major WPC processing methods (Clemons 2002). However, these conventional processing methods showed limitations in improving the performances of WPCs. Hence, the development of new processing methods has been highly required in WPCs and recently, as an innovative molding technology, co-extrusion technique has been introduced to WPCs (Kim et al. 2013; Huang et al. 2013). The use of co-extrusion processing in WPCs makes the new application of WPCs possible with enhanced performances and reduced costs.

This paper provides an overview for WPCs focusing on the use of bio-based plastics and co-extrusion technique.

\section{WOOD}

Wood filler is a porous and anisotropic material with showing different features among species (e.g., softwood and hardwood) (Maldas et al. 1989). For example, softwood fillers are more flexible compared to hardwood fillers. However, more importantly considered properties of wood fillers are size, shape, and aspect ratio (Xanthos 2005). Wood fillers can be acquired from by-products in wood industries (e.g., saw dust, chips, flakes, and recycled pulps, etc.) and utilized as received or after some mechanical and/or chemical processing to give particle, flour, and fiber shapes. The use of wood fillers in plastic composites are growing rapidly because the wood filler gives excellent properties such as light weight, dimensional stability, and high stiffness to filled plastics while keeping cost competitiveness (Yam et al. 1990; Wolcott and Englund 1999).

Wood particles have approximately equal sizes in all directions and the property improvements of filled plastic composites are not high enough except for their cost effectiveness. It was mainly utilized in the initial step of WPC development.

Wood flour is considered as an effective reinforcing filler for WPCs because it has a special characteristic bearing a majority of the applied load in filled plastic composites compared to wood particles (Matthews and Rawlings 1999). Hence, wood flour is preferred as one of the widely utilized wood fillers in WPCs. Wood flour has relatively high aspect ratio compared to wood particle and the incorporation of wood flour into plastic matrices leads to much improved strength and stiffness in filled WPCs (Stark and Berger 1997). Wood flours can be acquired from common species such as pine, maple, and oak on availability.

Wood pulp having longer fiber shapes can be divided into mechanically-processed pulp (MP) and chemically-processed pulp (CP). The MP still holds large amounts of lignin (e.g., $28.1 \%$ for Radiata pine and $27.89 \%$ for Douglas fir, respectively) (Iiyama and Wallis 1988; Boussaid and Saddler 1999). The MP from softwood has approximately 3-10 mm of length and below $0.05 \mathrm{~mm}$ of diameter showing over 100 of aspect ratios. On the other hand, CP features smaller bundles of more pure cellulose fibrils by dissolving the bonding lignin. Thus, CP generally shows the stronger mechanical property than MP (Woodhams et al. 1984). 


\section{TREATMENTS}

The limitations of wood fillers used in WPCs include 1) poor compatibility with hydrophobic plastics 2) high moisture uptake 3) low thermal stability during processing. Among these, the poor compatibility between wood surfaces and plastic matrices is a major issue in WPC developments because the interfacial bonding between them influences the mechanical properties of filled composites and also, the quality of this bonding affects the ability of transferring stresses from the matrix to the wood fillers and consequently determines the mechanical properties of the resultant composites (Faruk et al. 2012). Therefore, increasing the compatibility between wood and plastic is very important in improving the performances of WPCs. There are several coupling mechanisms to improve the compatibility between wood and plastic matrix. These approximately can be classified into two treatments: physical and chemical treatments. In this section, some surface treatments of wood fiber are reviewed.

\subsection{Physical Treatment}

Conventional physical treatments of wood fillers include simple drying and thermo-treatment methods. Drying is frequently carried out before main treatments. Drying method is preferred to prevent from the evaporation of water within wood fillers occurring during thermal compounding process, because the water evaporation gives rise to poor bonding or gap between wood fillers and polymer matrices leading to crack and fracture initiations. Thermotreatments are widely applied to wood fillers to give more hydrophobic characteristics through the thermal degradation of hemicelluloses and lignin rearrangements in no oxygen condition (Raj et al. 1990).

Steam explosion method uses higher pressure and temperature leading to morphological and structural changes of lignocellulosic materials in wood fillers (Park et al. 2004; Kim et al. 2012). Steam explosion treated wood fillers can be fractionized into respective components (i.e., cellulose, lignin, and hemicellulose) to a certain extent and this makes high percentages of celluloses in wood possible with increased surface area.

Plasma is a partially or fully ionized gas, which is called the fourth state of matter containing electrons, ions, and neutral atoms (Chang 1998). Plasma treatment is used for increasing the surface energy of materials by introducing the plasma to material surface. It modifies the surfaces via producing increased surface activity (e.g., reactive free radicals and groups) and these surface modifications can be achieved using various gases.

Corona treatment is similar to plasma treatment. Electro-magnetic field produced by corona generator modifies the surface energy of materials depending on voltage, air pressure, and sample geometry. It is normally generated by using a low temperature corona discharge and the corona treatment on wood filler and polymer matrix effectively lead to improved adhesions between wood and plastic matrix (Belgacem et al. 1994). This is because there are some changes in the morphology and the amounts of functional groups or effective cross-links in a region near the wood surface. Hence, the corona-treated surfaces show improved adhesion and compatibility to other polymer materials (Felix et al. 1994).

\subsection{Chemical Treatment}

Maleation is one of the most widely utilized coupling treatments to impart improved mechanical properties to WPCs. Numbers of researches have been performed to investigate the effect of maleic anhydride grafting on the physical, thermal, and me- 


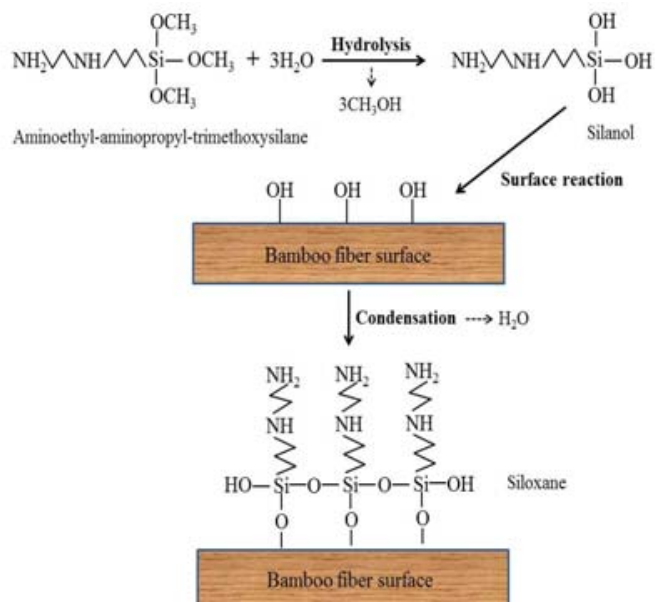

Fig. 1. Schematic diagram of bamboo fiber surfaces chemically modified by silane treatment (Kim et al. 2012).

chanical properties of wood fibers and filled plastic composites ( $\mathrm{Lu}$ and $\mathrm{Wu} 2000)$. For wood fibers, the maleation caused significant improvements in thermo-mechanical properties of WPCs through improved compatibility between treated wood surface and plastic matrix ( $\mathrm{Lu}$ et al. 2002). Also, as coupling agents, maleic anhydride grafted polymers led to enhanced adhesions between wood fiber and plastic matrix through ester linkages and hydrogen bonds between wood surface and anhydride groups in grafted polymers (Lu et al. 2005).

Silane has been recognized as one of the efficient coupling agents widely utilized in natural fiber/wood filled plastic composites (Xie et al. 2010). In most cases, the silane coupling agent is used for wood surface modifications. It undergoes 3 steps during the silane treatment process: hydrolysis, condensation and bond formation. After the hydrolysis of silane coupling agents, silanols are produced and then the condensation of the silanols is consecutively performed with the bond formations between filler and matrix (Lee et al. 2008; Kim et al. 2012). Fig. 1 shows a schematic diagram of the silanization proc- ess onto bamboo surfaces.

Alkali treatment by $\mathrm{NaOH}$ is utilized as a chemical treatment to remove mainly lignin and extractives in natural/wood fibers (Yue et al. 2012). The elimination of lignin and extractives (specially, waxy materials) at the surface of cellulose intra-crystalline and inter-crystalline regions of wood fibers by the alkali treatment provides more uniform and cleaner fiber surfaces with increased aspect ratio from reduced fiber diameter, leading to better fiber matrix interfacial adhesion and mechanical interlocking (Kabir et al. 2011). As a result, alkali treated fiber filled composites can show remarkably improved mechanical and thermal properties.

Besides, acetylation, peroxide, and enzyme treatments are used to treat wood fillers and nowadays, among them, the enzyme treatment attracts more attentions due to the increased concern for environmental contaminations, and its uses are accordingly being extended in industries.

\section{PLASTICS}

So far, plastic market has been dominated by petroleum-based plastics and WPCs are mainly produced using the petroleum-based thermoplastics such as polyethylene (PE), polypropylene (PP), and polyvinylchloride (PVC). However, government and public concerns for the environmental waste, climate change and limited fossil fuel resources let companies and researchers develop alternative biodegradable plastics to the conventional petroleum-based plastics. Now, bio-based plastics from renewable raw materials are receiving more attentions than past years (Mohanty et al. 2000). In this section, representative petroleum-based and bio-based thermoplastics used in the production of WPCs are introduced. 


\subsection{Petroleum-based Thermoplastics}

As plastic matrices for WPCs, petroleum-based thermoplastics such as PE, PP, and PVC has been widely utilized. PE is one of the most widely used plastics in the world now (Harper 1999). It has easy processing, good ductility, high toughness, and excellent chemical resistance. However, it has fundamental limitations in its relatively low modulus and yield stress. Hence, remarkably improved modulus and stress can be expected in the wood fiber filled PE composites (Kim 2012). PP is another thermoplastic polymer, which is synthesized by the polymerization of propylene monomer derived from petroleum products through reactions using catalysts. These catalysts allowed the control of stereochemistry during polymerization to form PP. As a result, PP shows more rigid and higher stiffness characteristics compared to PE. PVCs are prepared by the polymerization of vinyl chloride in a free-radical addition polymerization reaction. PVC features linear shapes with a low number of short-chain branches. Main disadvantages of PVC are brittleness and relatively low thermal decomposition temperature and therefore, to blend PVC with wood filler at elevated temperatures, incorporation of plasticizers or increased PVC gelation degree are required (Matuana et al. 1997; Lewandowski et al. 2011).

Besides, nylon is frequently used to make WPCs, because it shows relatively high mechanical properties (e.g., strength, modulus, and impact) compared to general plastics. Crystalline characteristics from its special structure having the amide linkage in its backbone and low friction coefficient with good abrasion resistance have received attentions (Berins 2002), but relatively high melting temperature of nylon (normally, over $200^{\circ} \mathrm{C}$ ) prevents its further uses in WPC manufacturing because filled wood fibers are thermally decomposed.

\subsection{Bio-based Thermoplastics}

Bio-based plastics mentioned in here include biodegradable thermoplastics such as polylactide or polylactic acid (PLA) derived from renewable feedstock and polyhydroxybutyrate (PHB) and polyhydroxybutyrate-co-valerate (PHBV) produced from bacteria-aided biochemical processing. Nowadays, the bio-based plastics are receiving increased attentions from the industries and manufacturers seeking to replace petroleum-based polymers with biodegradable materials and the technological development of bio-based plastics is helping it (Mohanty et al. 2002).

Polylactide or polylactic acid (PLA) is a thermoplastic polyester synthesized from the ring opening process of lactides, which are cyclic diesters of lactic acid from fermentable sugar (Brydson 1999) or by direct condensation polymerization of lactic acid (Ajioka et al. 1995). It is now one of the most preferably and widely utilized bio-based thermoplastics in market and the application of PLA into wood/natural fiber filled plastic composites are being remarkably extended (Mohanty et al. 2000).

Polyhydroxybutyrate (PHB) and polyhydroxybutyrate-co-valerate (PHBV) are used in the field of wood/ natural fiber filled bio-composites as another biodegradable plastics (Zaverl et al. 2012). These plastics are produced through biochemical processes using bacteria, which is different from typical plastic manufacturing methods. In the case of PHB, when the bacteria feed glucose, it is produced. On the other hand, for PHBV, when the bacteria feed the mixture of glucose and propionic acid, it is produced (Mohanty et al. 2000).

Since bio-based plastics blended with wood/natural fiber were introduced to WPC industries, conventional polyolefin-based WPCs are being replaced with these fully degradable bio-composites (Mohanty 
et al. 2000; Mohanty et al. 2002). However, cost increases from the use of bio-based plastics are preventing further applications.

\section{PROCESSING METHODS}

Normally, WPC compositions (i.e., wood, plastic, and additives) are compounded at $20 \sim 30^{\circ} \mathrm{C}$ over melting temperatures of base plastic matrices by using a conventional plastic product manufacturing method. After compounding, respective blends or pellets are prepared and then molded to have a shape for target applications by using extrusion, injection, and compression molding equipment. These processing techniques have been used as confirmed manufacturing methods for producing WPC products with controllable quality. In these days, the development of technique and accumulation of experience make the application of new manufacturing methods for WPCs possible. Structured WPCs produced with innovative technology (i.e., co-extrusion) have a lot of potentials for new engineering and advanced applications. In this section, major molding methods used in manufacturing WPC products are reviewed with the introduction of co-extrusion manufacturing method.

\subsection{Compounding Process}

Compounding processes can be described as blending processes which involve mixing, kneading, and shearing before manufacturing products. Hence, in WPCs, the compounding process is normally carried out to blend WPC compositions. Blended and compounded ingredients are formed into pellets for future processing. For equipment, $\mathrm{CW}$ Brabender mixers using internal shear forces or mills such as Gelimat or Szego mills have been used to compound the WPC compositions (Matuana et al. 1997). However, in these days, extruders with a single-screw or twin-screws are preferred due to their better compounding capability.

\subsection{Extrusion Process}

Extrusion processes are utilized in not only compounding or manufacturing pellets/granules, but also directly producing WPC products by using direct extrusion methods (e.g., inline-manufacturing). Singleand twin-screw extruders are representative extrusion equipment and generally, twin-screw extruders are preferred because they show better compounding and extruding effects compared to single-screw extruders. The operation methods (e.g., rotating, co-rotating and counter-rotating) of extruders affect resultant compounding and extruding qualities. Through the use of co- or counter-rotating methods, more homogeneously dispersed wood fillers can be obtained in the molten plastic matrices (Kim et al. 2009). Also, the properties of extruded WPCs are affected by the extrusion processing parameters (e.g., extruding time, screw rpm, and barrel temperature, etc.) (Takase and Shiraishi 1989; Saheb and Jog 1999).

Decking, fencing, and railings produced by using a profile extrusion method comprise large amounts of WPC market (Clemons 2002). Manufacturing steps include firstly compounding process together with wood, plastic, and additives and then the compounded molten materials are forced through a die to have a continuous WPC profile. The shapes of the extruded WPC profiles can be a designed from simple solid to hollow structure (Rowell 2005).

\subsection{Injection Molding}

Injection molding is one of the preferred manufacturing methods in WPC industries due to the fact that it can produce a variety of complex geometric 
products with fast and functional fashion. Thus, although the total poundage of injection-molded WPC products is less than that of extruded WPCs, the injection molding method is a good choice when the continuous piece is not desired or more complicated shapes are needed. However, in formulation, wood contents should be adjusted because high amount of wood filler can lead to increased viscosity preventing injection molding processes (Rowell 2005). The injection molding method uses a screw as a transport tool. The screw geometry in the injection molding equipment is important because it performs nearly every step of injection molding processes such as blending, melting, conveying, and pumping.

\subsection{Compression Molding}

Compression molding method can be used in manufacturing of WPCs for both thermo-plastics and thermo-sets. Compared to extrusion- and injection molding methods, the compression-molding method makes bigger sizes or volumes of WPC products possible. Hence, in automotive or construction companies requiring bigger panels or parts for interior or exterior uses, compression molding method is preferred (Drzal et al. 2001). Compression molding methods used in the WPC product manufacturing follow next steps: 1) compounded and extruded WPC pellets are put into a mold and the mold is heated to the determined temperature 2) enough load is applied to the molten WPC materials by bringing the two halves of the molds 3) pressure is exerted to squeeze the molten WPC materials so that it fills the mold cavity 4) after a certain time is passed, resultant compression-molded WPC products can be acquired. However, the compression molding method has some disadvantages such as relatively low productivity from longer cycle time and high cost to produce bigger molders.

\subsection{Co-extrusion Process}

Extrusion, injection, and compression molding methods have been utilized as conventional WPC manufacturing techniques. WPC products manufactured by using these conventional molding methods have comprised most part in WPC market. However, WPC products adopting conventional methods have relatively low properties compared to other functional fillers (e.g., glass fiber and carbon fiber) reinforced plastic composites. Hence, a development of new type of WPC manufacturing method is highly required. Also, in recent years, most WPC companies are facing to cost increase and strengthened environment regulations. Hence, approaches maintaining cost effectiveness with solving environmental problems are very important in developing WPC products. As one approach, some companies have considered the use of recycled plastics as base polymer systems for WPC products, but this approach essentially led to property decreases. Therefore, a new processing technique to meet the above whole requirements was introduced in the field of WPCs.

Co-extrusion technique consists of two or more extruders combined with one die to produce multiple-layered products. By combining molten multiple plastic layers with various properties into one profile, optimized product performances can be achieved (Rosato 1998). Improved water proof and oxygen barrier property with toughness increases are advantages of co-extruded products (Rosato 1998; Giles et al. 2005). So far, this co-extrusion technique has been mostly applied to produce packaging films for maintaining content freshness or pipes requiring high mechanical properties (Kim et al. 1984; Doshi et al. 1988). Recently, WPCs using the co-extrusion technique has been developed. Fig. 2 shows the pilot scale co-extrusion WPC manufacturing system developed by Prof. Qinglin Wu's lab of LSU Agcenter in 


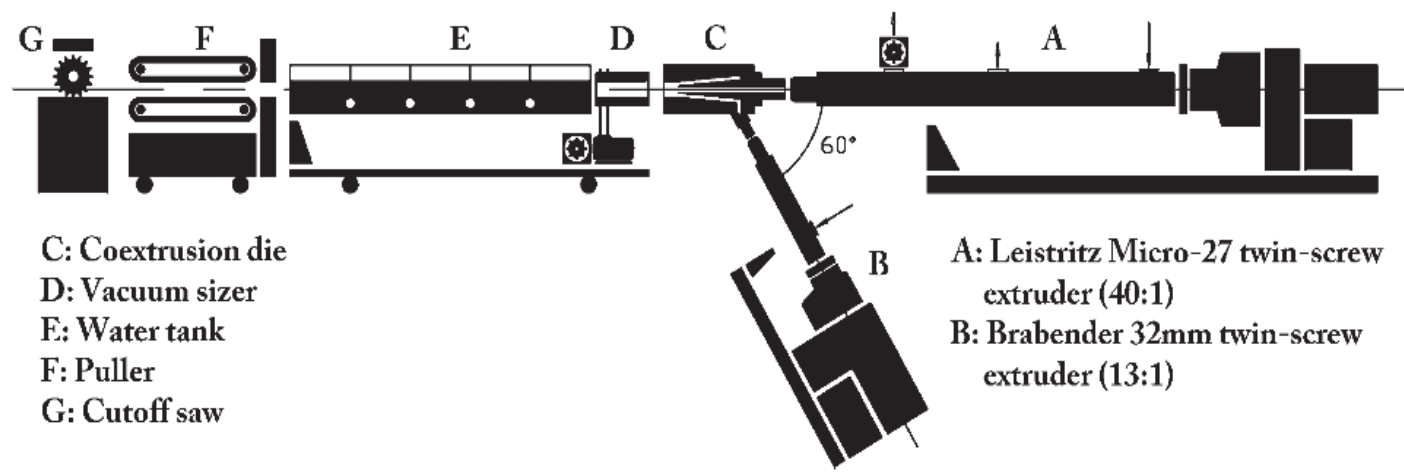

Fig. 2. Schematic diagram of the pilot scale co-extrusion WPC manufacturing system (Yao and Wu 2010).

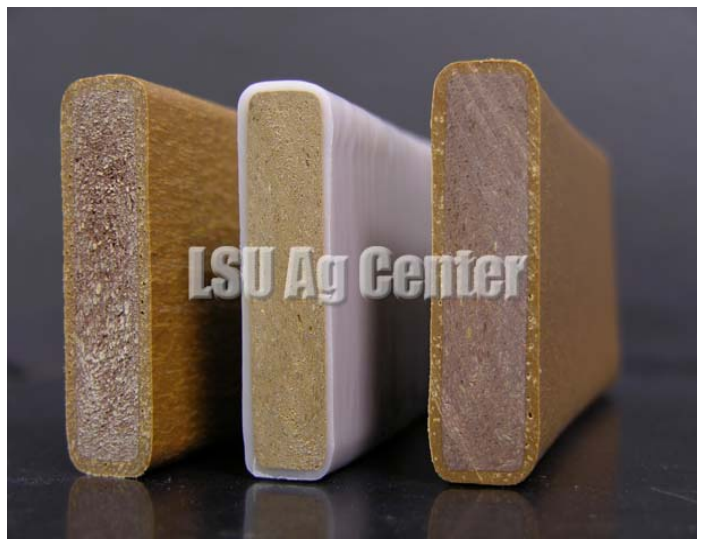

Fig. 3. Co-extruded WPC profiles produced by co-extrusion manufacturing system (LSU Ag Center).

USA and co-extruded WPC profiles produced by using this system are also observed in Fig. 3.

As shown in the above figure, the manufactured co-extruded WPC profiles show core-shell structured shapes to fundamentally enhance WPC performances (Kim 2012). By proper combination of constituting layers, one can achieve a balance of such properties as light weight, high strength, high stiffness, wear resistance, biological resistance, unusual thermal expansion characteristics, appearance, and controllable biodegradability (Yao and $\mathrm{Wu}$ 2010; Kim et al. 2013; Huang et al. 2013).

\section{CONCLUSION}

Wood fillers such as wood particle, wood flour, and wood pulp with different size, shape, and aspect ratio have been used as reinforcing fillers for WPCs due to good properties (e.g., light weight, dimensional stability, high stiffness, and low cost). However, hydrophilic characteristics of wood fillers need surface modifications to effectively couple with hydrophobic plastics. To improve interfacial adhesions between wood and plastic matrix leading to enhanced performances, numbers of researchers and industries have attempted various physical (drying, steam explosion, plasma/corona, etc.) and chemical (maleation, silanization, alkali, etc.) treatments. Although the improved WPC performances through these treatments have extended WPC market by manufacturing reliable WPC products, increased environmental concerns have required the use of new green plastic materials (i.e., bio-based plastics) in WPCs. Now, bio-based plastics such as polylactide and polyhydroxybutyrate are being applied to WPCs, but relatively expensive price of existing biodegradable plastics prevent further uses. Current WPC processing methods (i.e., extrusion, injection, and compression moldings) have showed reliable product 
performances. However, there are still some limitations to use the WPC products in engineered or structural applications. Hence, a new processing technique (i.e., co-extrusion method) has been introduced to WPCs and by using this co-extrusion system, core-shell structured WPCs were manufactured.

So far, various treatments, plastics, and processing techniques have been applied to WPC industries and showed some improvements in product performances. However, further developments are still needed for WPCs and these will include structured WPCs having highly tunable layers with the use of biodegradable/bio-based plastic materials.

\section{ACKNOWLEDGEMENT}

This study was carried out with the support of 'Forest Science \& Technology Projects (Project No. S211314L010140)' provided by Korea Forest Service.

\section{REFERENCES}

Ajioka, M., Enomoto, K., Suzuki, K., Yamaguchi, A. 1995. The basic properties of poly(lactic acid) produced by the direct condensation polymerization of lactic acid. J. of Environmental Polymer Degradation, 3(4): 225 234.

Belgacem, M.N., Bataille, P., Sapieha, S. 1994. Effect of corona modification on the mechanical properties of polypropylene/cellulose composites. J. of Applied Polymer Science, 53(4): 379 386.

Berins, M.L. 2002. Plastics Engineering Handbook of the Society of the Plastics Industry, Kluwer Academic Publishers, Norwell, USA.

Bledzki, A K., Reihmane, S., Gassan, J. 1998. Thermoplastics reinforced with wood fillers: A literature review. J. Polymer-Plastics Technology and Engineering 37(4): 451 468.

Borysiak, S. 2013. Fundamental studies on ligno- cellulose/polypropylene composites: Effects of wood treatment on the transcrystalline morphology and mechanical properties, J. of Applied Polymer Science 127(2): 1309 1322.

Boussaid, A., Saddler, J.N. 1999. Adsorption and activity profiles of cellulases during the hydrolysis of two Douglas fir pulps. Enzyme and Microbial Technology, 24(3-4): 138 243.

Brydson, J.A. 1999. Plastics Materials, Butterworth Heinemann Publishing, Woburn, USA.

Chang, T.C. 1998. Plasma surface treatment in composites manufacturing. J. of Industrial Technology, 15(1): $2 \sim 7$.

Clemons, C. 2002. Wood-plastic composites in the United States - The interfacing of two industries. J. of Forest Products 52(6): 10 18.

Doshi, S.R., Charrier, J.M., Dealy, J.M. 1988. A coextrusion process for the manufacture of short-fiberreinforced thermoplastic pipe. Polymer Engineering and Science, 28(15), 964 973.

Drzal, L.T., Mohanty, A., Misra, M. 2001. Bio-composite materials as alternatives to petroleumbased composites for automotive applications. In Proc. of Automotive Composites Conference, pp. $1 \sim 8$. Troy, USA.

Faruk, O., Bledzki, A.K., Fink, H.-P., Sain, M. 2012. Biocomposites reinforced with natural fibers: 2000-2010. Progress in Polymer Science, 37(11): $1552 \sim 1596$.

Felix, J., Gatenholm, P., Schreiber, H.P. 1994. Plasma modification of cellulose fibers: Effects on some polymer composite properties. J. of Applied Polymer Science, 51(2): 285 295.

Giles, H.F., Wagner, J.R., Mount, E.M. 2005. Extrusion: The definitive processing guide and handbook, William Andrew Inc., Norwich, USA.

Harper, A. 1999. Modern Plastics Handbook, McGrawHill, New York, USA.

Hoadley, R.M. 1990. Identifying Wood - Accurate 
Results with Simple Tools, Taunton Press, Connecticut, USA.

Huang R., Kim, B.-J., Lee, S., Yang, Z., Wu, Q. 2013. Co-Extruded Wood-Plastic Composites with Talc-Filled Shells: Morphology, Mechanical, and Thermal Expansion Performance. Bioresources 8(2): $2283 \sim 2299$.

Iiyama, K., Wallis, A.F.A. 1988. An improved acetyl bromide procedure for determining lignin in woods and wood pulps. Wood Science and Technology, 22(3): 271 280.

John, M.J., Thomas, S. Biofibres and biocomposites. 2008. Carbohydrate Polymers 71(3): 343 364 .

Kabir, M.M., Wang, H., Aravinthan, T., Cardona, F., Lau, K.-T. 2011. Effects of natural fibre surface on composite properties: A review. In Proc. of 1st International Postgraduate Conference on Engineering, Designing and Developing the Built Environment for Sustainable. pp. 94 99, Wellbeing, Australia.

Kim, B.-J. 2012. The effect of inorganic fillers on the properties of wood plastic composites. Ph.D. Dissertation, Louisiana State University, USA.

Kim, B.-J. Yao, F., Han, G., Wang, Q., Wu, Q. 2013. Mechanical and physical properties of core-shell structured wood plastic composites: Effect of shells with hybrid mineral and wood fillers. Composites Part B: Engineering 45(1): $81 \sim 84$.

Kim, B.-J., Yao, F., Han, G., Wu, Q. 2012. Performance of bamboo plastic composites with hybrid bamboo and precipitated calcium carbonate fillers. Polymer Composites, 33(1): $68 \sim 78$.

Kim, S., Do, I., Drzal, L.T. 2009. Multifunctional $\mathrm{xGnP} /$ LLDPE nanocomposites fabricated by solution compounding method and various screw rotating systems. Macromolecular Materials and Engineering, 294(3): 196 205.

Kim, Y.J., Han, C.D., Song, B.K., Kouassi, E. 1984.
Mechanical and transport properties of coextruded films. Journal of Applied Polymer Science 29(7): 2359 2382.

Lee, S.-Y., Kang, I.-A., Doh, D.-H., Yoon, H.-G., Park, B.-D., Wu, Q. 2008. Thermal and mechanical properties of wood flour/talc-filled polyactic acid composites: Effect of filler content and coupling treatment. J. of Thermoplastic Composite Materials, 21(3): 209 223.

Lewandowski, K., Zajchowski, S., Mirowski, J., Kościuszko, 2011. A. Study of processing properties of PVC/wood composites. CHEMIK International, 65(4): 329 336.

Lu, J.Z., Negulescu, I.I., Wu, Q. 2002. Thermal and dynamic-mechanical properties of wood-PVC composites. in Proc. of 6th Pacific Rim Bio-Based Composites Symposium \& Workshop on the Chemical Modification of Cellulosics, pp. 103 111, Portland, USA.

Lu, J.Z., Negulescu, I I., Wu, Q. 2005. Wood-fiber/ high-density-polyethylene composites: Coupling agent performance. J. of Applied Polymer Science, 96(1): $93 \sim 102$.

Lu, J.Z., Wu, Q. 2000. Chemical coupling in wood fiber and polymer composites: A review of coupling agents and treatments. Wood and Fiber Science, 32(1), 88 104.

Mahlberg, R., Niemi, H.E.-M., Denes, F.S., Rowell, R. M. 1999. Application of AFM on the Adhesion Studies of Oxygen-Plasma-Treated Propylene and Lignocellulosics, Langmuir, 15(8): 2985 2992.

Maldas, D., Kokta, B.V., Daneault, C. 1989. Composites of polyvinyl chloride-wood fibers: IV. Effect of the nature of fibers. J. of Vinyl \& Additive Technology, 11(2): 90 99.

Matthews, F.L., Rawlings, R.D. 1999. Composite Materials: Engineering and Science, Woodhead Publishing Limited, Sawston, UK.

Matuana, L.M., Park, C.B., Balatinecz, J.J. 1997. 
Overview of Wood Plastic Composites: Focusing on Use of Bio-based Plastics and Co-extrusion Technique

Processing and cell morphology relationships for microcellular foamed PVC/wood-fiber composites. Polymer Engineering and Science, 37(7): 1137 1147.

Matuana, L.M., Park, C.B., Balatinecz, J.J. 1997. The effect of low levels or plasticizer on the rheological and mechanical properties of polyvinyl chloride/newsprint-fiber composites. J. of Vinly \& Additive Technology, 3(4): 265 273.

Mohanty, A.K., Misra, M., Drzal, L.T. 2002. Sustainable bio-composites from renewable resources: Opportunities and challenges in the green materials world. J. of Polymers and the Environment 10(1): 19 26.

Mohanty, A.K., Misra, M., Hinrichsen, G.. 2000. Biofibres, biodegradable polymers and biocomposites: An overview. Macromolecular Materials and Engineering 276(1): 1 24.

Park, B.-D., Wi, S.G.., Lee, K.H., Singh, A.P., Yoon, T.-H., Kim, Y.S. 2004. X-ray photoelectron spectroscopy of rice husk surface modified with maleated polypropylene and silane. Biomass and Bioenergy, 27(4): 353 363.

Raj, R.G.., Kokta, B.V., Daneault, C. 1990. A comparative study on the effect of aging on mechanical properties of LLDPE-glass fiber, mica, and wood fiber composites. Polymer Engineering \& Science, 4(5): $645 \sim 655$.

Rosato, V. 1998. Extruding plastics - A practical processing handbook, Chapman \& Hall, Norwell, USA.

Rowell, R.M. 2005. Handbook of Wood Chemistry and Wood Composites, CRC Press, Boca Raton, USA.

Saheb, N., Jog, J.P. 1999. Natural fiber polymer composites: A review. Advances in Polymer Technology, 18(4): 351 363 .

Stark, N.M., Berger, M.J. 1997. Effect of Particle
Size on Properties of Wood-Flour Reinforced Polypropylene Composites. In Proc. of 4th International Conference on Woodfiber-Plastic Composites, pp.134 143, Madison, USA.

Takase, S., Shiraishi, N. 1989. Studies on composites from wood and polypropylene. II. Journal of Applied Polymer Science, 37(3): 645 659.

Wolcott, M.P., Englund, K. 1999. A technology review of wood-plastic composites. In Proc. of 33rd International Particleboard/Composite Materials Symposium, pp. 103 111, Pullman, USA. Woodhams, R.T., Thomas, G., Rodgers, D.K. 1984. Wood fibers as reinforcing fillers for polyolefins. Polymer Engineering \& Science, 24(15): 1166 1171.

Xanthos, M. 2005. Functional Fillers for Plastics, Wiley-VCH, Weinheim, Germany.

Xie, Y., Hill, C.A.S., Xiao, Z., Militz, H., Mai, C. 2010. Silane coupling agents used for natural fiber/polymer composites: A review. Composites Part A: Applied Science and Manufacturing, 41(7), 806 819.

Yam, K.L., Gogoi, B.K., Lai, C.C., Selke, S.E. 1990. Composites from compounding wood fibers with recycled high density polyethylene. Polymer Engineering \& Science, 30(11): 693 699 .

Yao, F., Wu, Q. 2010. Coextruded polyethylene and wood-flour composite: Effect of shell thickness, wood loading, and core quality. Journal of Applied Polymer Science, 118(6): 3594 3601.

Yue, Y., Zhou, C., French, A.D., Xia, G., Han, G., Wang, Q., Wu, Q. 2012. Comparative properties of cellulose nano-crystals from native and mercerized fibers. Cellulose, 19(4): 1173 1187.

Zaverl, M., Seydibeyoglu, M.Ö., Misra, M., Mohanty, A. 2012. Studies on recyclability of polyhydroxybutyrate-co-valerate bioplastic: Multiple melt processing and performance evaluations. J. of Applied Polymer Science, 125(2): 324 331. 